\title{
New injection scheme using a pulsed quadrupole magnet in electron storage rings
}

\author{
Kentaro Harada, ${ }^{*}$ Yukinori Kobayashi, Tsukasa Miyajima, and Shinya Nagahashi \\ Photon Factory, High Energy Accelerator Research Organization, 1-1 Oho, Tsukuba, Ibaraki 305-0801, Japan
}

(Received 30 July 2007; published 21 December 2007)

\begin{abstract}
We demonstrated a new injection scheme using a single pulsed quadrupole magnet (PQM) with no pulsed local bump at the Photon Factory Advanced Ring (PF-AR) in High Energy Accelerator Research Organization (KEK). The scheme employs the basic property of a quadrupole magnet, that the field at the center is zero, and nonzero elsewhere. The amplitude of coherent betatron oscillation of the injected beam is effectively reduced by the PQM; then, the injected beam is captured into the ring without largely affecting the already stored beam. In order to investigate the performance of the scheme with a real beam, we built the PQM providing a higher field gradient over $3 \mathrm{~T} / \mathrm{m}$ and a shorter pulse width of $2.4 \mu \mathrm{s}$, which is twice the revolution period of the PF-AR. After the field measurements confirmed the PQM specifications, we installed it into the ring. Then, we conducted the experiment using a real beam and consequently succeeded in storing the beam current of more than $60 \mathrm{~mA}$ at the PF-AR. This is the first successful beam injection using a single PQM in electron storage rings.
\end{abstract}

DOI: 10.1103/PhysRevSTAB.10.123501

PACS numbers: 07.55.Db, 29.20.Dh, 29.27.Ac, 41.60.Ap

\section{INTRODUCTION}

In a conventional injection scheme for electron storage rings such as synchrotron light sources or electron-positron colliders, several pulsed dipole magnets, which are called kicker magnets, are employed to reduce the effective amplitude of the injected beam and facilitate beam injection while preserving an already stored beam [1]. Such a scheme enables us to accumulate the electron beam in the storage ring by utilizing the radiation damping characteristics of the electron beam. The beam injected into the ring is instantaneously perturbed by the kickers located downstream from an injection point. Simultaneously, the kickers are turned off and the beam executes betatron oscillation. It is essential that the kickers are operated at a speed of the order of several microseconds. Subsequently, the beam is captured in the acceptance of the ring and it is damped down to the equilibrium emittance by radiation damping. On the other hand, the stored beam with the equilibrium emittance passes a pulsed local bump, which is formed by the kickers around the injection point; the beam preserves its central orbit in other parts of the ring. This is repeated at a rate of several hertz or several tens of hertz. Figure 1 shows a schematic diagram of a conventional injection scheme using a pulsed local bump with four kickers.

However, it is difficult to make a complete closed bump for the stored beam because of field errors, timing jitters, individual differences of the kickers, and nonlinear elements like sextupole magnets inside the bump. These factors produce a leakage of the bump and generate coherent dipole oscillations in the stored beam during the injection time. Usually, this problem was not very serious since the beam shutters are closed and the experiments stopped

\footnotetext{
*kentaro.harada@kek.jp
}

in most synchrotron light sources. However, in light sources operating in top-up mode (continual injection with shutters open), the oscillations are a serious problem because the intensity of a photon beam is modulated by the oscillations. In order to solve the problem, it has been very important to suppress the coherent dipole oscillations produced by the leakage of the bump [2].

The PF-AR is a unique synchrotron radiation light source dedicated to single-bunch operation for a pulsed $\mathrm{x}$ ray. It was originally constructed as a booster synchrotron for the TRISTAN, which was an electron-positron collider. Since 1997, the PF-AR has dedicatedly delivered the synchrotron radiation, finishing the role as a booster [3]. In the upgrade carried out in 2001, the vacuum system was significantly improved. As a result, the beam lifetime increased to about 10 times its previous lifetime and now exceeds $15 \mathrm{~h}$ at an initial current of $60 \mathrm{~mA}$. An electron beam is injected at a lower energy of $3.0 \mathrm{GeV}$ and ramped up to $6.5 \mathrm{GeV}$. However, the lower energy injection causes

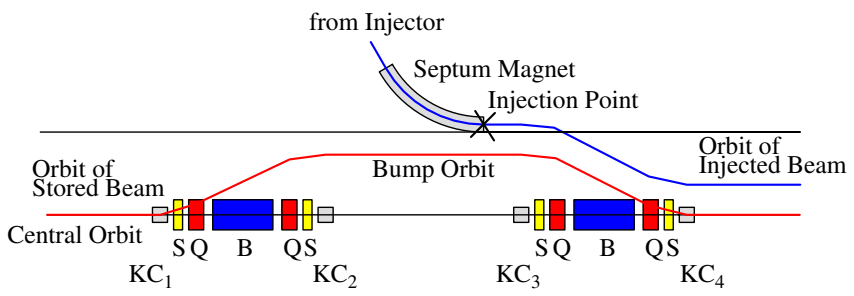

FIG. 1. (Color) Schematic drawing of a conventional injection scheme. After the injected beam is bent into the orbit by the septum magnet, the injected beam is perturbed by two kicker magnets $\mathrm{KC}_{3}$ and $\mathrm{KC}_{4}$; it then oscillates with a large amplitude in the ring. For the stored beam, the pulsed bump orbit is produced by four kicker magnets $K C_{1}, K C_{2}, K C_{3}$, and $K C_{4}$. $\mathrm{B}, \mathrm{Q}$, and $\mathrm{S}$ denote the bending, quadrupole, and sextupole magnets, respectively. The cross represents the injection point. 
some difficulties in storing a higher beam current even in single-bunch operation. Normal conducting multicell rf cavities with an alternating periodic structure were adopted to realize a higher accelerating voltage of $3 \mathrm{MV}$ in short straight sections [4]. The multicell-type cavities have many higher order modes, which cause dangerous beam instabilities at a higher beam current. A delicate tuning of the rf voltage is essential to avoid the instabilities. In addition, a transverse bunch feedback system and octupole magnets are also required to suppress the transverse instabilities during beam injection. Much effort was devoted to accomplish storing the beam up to a current of more than $60 \mathrm{~mA}$.

For the injection in the PF-AR, we introduce an unclosed-bump technique. Since the septum magnets of the PF-AR are placed outside of the vacuum chamber for the storage ring, an injection point is somewhat distant from the central orbit of the stored beam. The injection position is about $50 \mathrm{~mm}$ away from the beam axis, and this value is 2 or 3 times larger than that of recent thirdgeneration synchrotron radiation sources. The unclosed local bump is obtained by providing stronger kicks to the kickers downstream from the injection point. This allows us to further reduce the amplitude of the oscillations in the injected beam compared with using a closed bump. The result is a coherent dipole oscillation in the stored beam due to an intentional leakage of the bump. When the transverse and/or the longitudinal instabilities are generated by the rf cavities at a higher beam current and the horizontal beam size increases, a part of the stored beam is lost at the vacuum chamber around the injection point due to the pulsed bump. For the operation of the PF-AR, it is very important to balance the amplitude reduction of the injected beam with the amplitude of the oscillation produced in the stored beam. If we can inject a beam without producing the pulsed local bump in the stored beam, we may easily accumulate the beam up to a much higher beam current [5]. For general top-up operation and resolving the problem in the PF-AR, we have promoted a new injection scheme using the single PQM so that no local bump is generated in electron storage rings [6]. Note that by adding a PQM the stored beam will suffer a beam size modulation after the injection process.

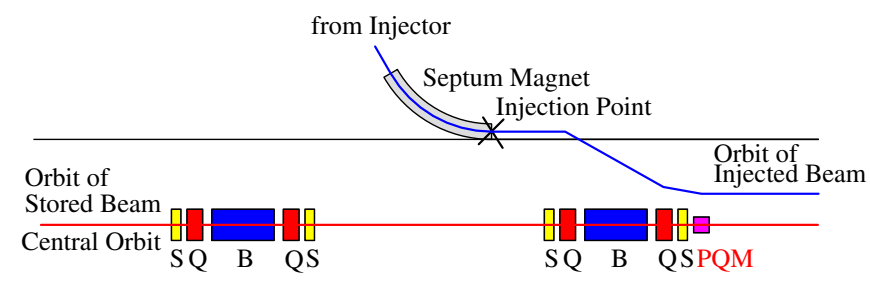

FIG. 2. (Color) Same as Fig. 1, but the injection scheme is using the PQM instead of the four kicker magnets. The injected beam is perturbed by the PQM; it then oscillates with a large amplitude in the ring. The stored beam passes through the central position of the PQM and its orbit is preserved on a central orbit.
In this paper, since the PF-AR injection optics is not standard in recent light sources, it is easier to explain the PQM injection scheme with a conventional optics layout first (i.e. shown in Fig. 2). Then, we apply the scheme to the special case of PF-AR in order to obtain the optimized strength of the PQM and confirm it using a multiparticle tracking simulation. Next, we describe the hardware of the PQM system to realize beam injection. Finally, we report the experimental result using a real beam.

\section{FORMULATION OF THE INJECTION SCHEME USING A SINGLE PQM}

\section{A. Conventional injection scheme using a pulsed local bump}

The beam, which comes from an injector, is injected into an electron storage ring after being bent into an orbit by a septum magnet, as shown in Fig. 1. The exit of the septum magnet is referred to as the injection point of the ring. The injection point is outside of the septum wall and horizontally far from the central orbit of the ring. The injected beam executes coherent betatron oscillation with large amplitude. In general, the betatron oscillation is affected by the magnetic nonlinearities of the ring. Here, for simplicity, we assume that nonlinearities are negligible and the betatron oscillation is linear. In addition, the beam is treated as a single particle. Then, the betatron oscillation of the beam follows an ellipse in the physical phase space, which is expressed by

$$
W_{i}=\frac{1}{\beta_{x, i}}\left\{x_{i}^{2}+\left(\alpha_{x, i} x_{i}+\beta_{x, i} x_{i}^{\prime}\right)^{2}\right\}=X_{i}^{2}+P_{i}^{2},
$$

where $x_{i}$ is the position, $x_{i}^{\prime}$ is the divergence angle, and $\left(x_{i}, x_{i}^{\prime}\right)$ denote the coordinates of the betatron oscillation at an injection point. Here, $\alpha_{x, i}$ and $\beta_{x, i}$ represent Twiss parameters in a horizontal direction, and $\left(X_{i}, P_{i}\right)$ are the normalized phase-space coordinates, and each of their elements is expressed by

$$
X_{i}=\frac{x_{i}}{\sqrt{\beta_{x, i}}}, \quad P_{i}=\frac{\alpha_{x, i} x_{i}+\beta_{x, i} x_{i}^{\prime}}{\sqrt{\beta_{x, i}}},
$$

where we call the ellipse $W_{i}$ an injection invariant.

Since the injection invariant without a perturbation to the beam is larger than the horizontal acceptance of the ring, we must reduce the invariant to facilitate beam injection. In order to achieve this, the kicker magnets are usually employed to produce a pulsed local bump. A reduced injection invariant using the local bump is represented by

$$
W_{1}=\frac{1}{\beta_{x}}\left\{\Delta x^{2}+\left(\alpha_{x, i} \Delta x+\beta_{x, i} \Delta x^{\prime}\right)^{2}\right\}=\Delta X^{2}+\Delta P^{2},
$$

where $\Delta x=x_{i}-x_{b}$ and $\Delta x^{\prime}=x_{i}^{\prime}-x_{b}^{\prime}$ denote the differences between the coordinates of the injection point and the coordinates of the pulsed bump, which are given by 
$\left(x_{b}, x_{b}^{\prime}\right)$. Here, the invariant with subscript 1 indicates the invariant for the injection beam after the bump. Then, the differences of the normalized coordinates, $(\Delta X, \Delta P)$, are represented by

$$
\left\{\begin{array}{l}
\Delta X \quad=X_{i}-X_{b}=\frac{\Delta x}{\sqrt{\beta_{x, i}}}, \\
\Delta P \quad=P_{i}-P_{b}=\frac{\alpha_{x, i} \Delta x+\beta_{x, i} \Delta x^{\prime}}{\sqrt{\beta_{x, i}}},
\end{array}\right.
$$

where $\left(X_{b}, P_{b}\right)$ are the normalized coordinates of the pulsed local bump at the injection point. Figure 3 displays the injection invariant on the normalized phase space of the betatron oscillations using the local bump. When the injection position $x_{i}$ and the bump position $x_{b}$ are given, $W_{1}$ is minimized by the condition $\Delta P=0$, i.e. $\Delta x^{\prime}=$ $-\left(\alpha_{x, i} / \beta_{x, i}\right) \Delta x$. The optimum $W_{1}$ is followed by

$$
W_{1}=\Delta X^{2}=\frac{\Delta x^{2}}{\beta_{x, i}}=\frac{\left(x_{i}-x_{b}\right)^{2}}{\beta_{x, i}},
$$

where the bump angle is

$$
x_{b}^{\prime}=x_{i}^{\prime}+\frac{\alpha_{x, i}}{\beta_{x, i}}\left(x_{i}-x_{b}\right) .
$$

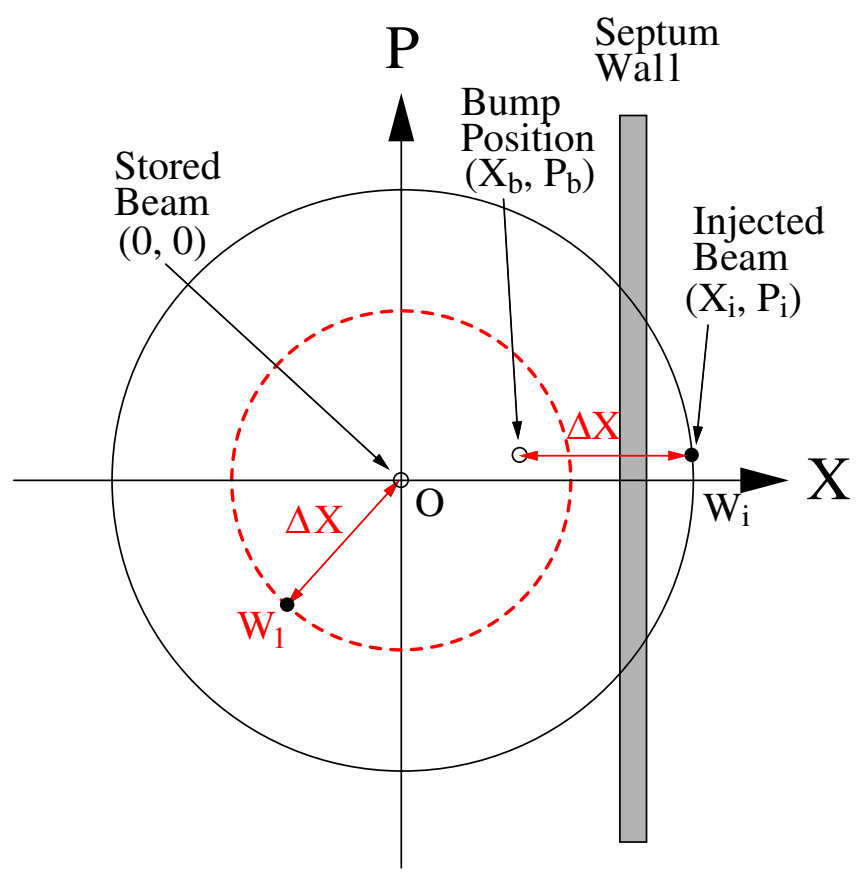

FIG. 3. (Color) Trajectories of the injected and stored beams using a pulsed local bump are displayed in the normalized phase space. The closed and open circles denote the injected and stored beams, respectively. $\left(X_{i}, P_{i}\right)$ are the coordinates of the injected beam and $\left(X_{b}, P_{b}\right)$ are the coordinates of the bumped orbit of the stored beam at the injection point. $\Delta X$ is the difference between the injection position and the bump position. $W_{i}$ and $W_{1}$ indicate the injection invariant and the reduced invariant, respectively. The rectangle shows the septum wall as a horizontal physical aperture.
In other words, the reduced invariant $W_{1}$ is obtained by dividing the difference between the injection and bump positions by the horizontal betatron function at the injection point. In order to obtain a smaller reduced invariant using the local bump, it is essential to use a thin septum wall and move the stored beam as close to the wall as possible. A larger betatron function at the injection point also allows us to obtain a smaller reduced invariant. However, we must consider the beam size of the stored beam to determine the height of the bump so that the beam is not lost by the septum wall.

Next, we explain the conventional injection scheme from the viewpoint of deriving the kick angle of the kickers. Figure 4 shows the trajectories of both the injected and stored beams using four kickers from $K C_{1}$ to $K C_{4}$ in the normalized phase space. The stored beam is at the origin of the coordinate axes, the central orbit. The beam passes through the bump coordinate of $\left(X_{b}, P_{b}\right)$ because of the upstream kickers $K C_{1}$ and $K C_{2}$, and then returns to the origin because of the downstream kickers $K_{3}$ and $K C_{4}$. The trajectory drawn by the dotted line indicates the closed bump of the stored beam. If we did not have the upstream kickers, the stored beam would be kicked by a large amount and may be lost from the acceptance of the ring. Thus, it is essential for the stored beam to form the local bump around the injection point.

On the other hand, the injected beam is at $\left(X_{i}, P_{i}\right)$, which are the injection coordinates. The beam is perturbed by the downstream kickers $K C_{3}$ and $K C_{4}$, and then reaches the

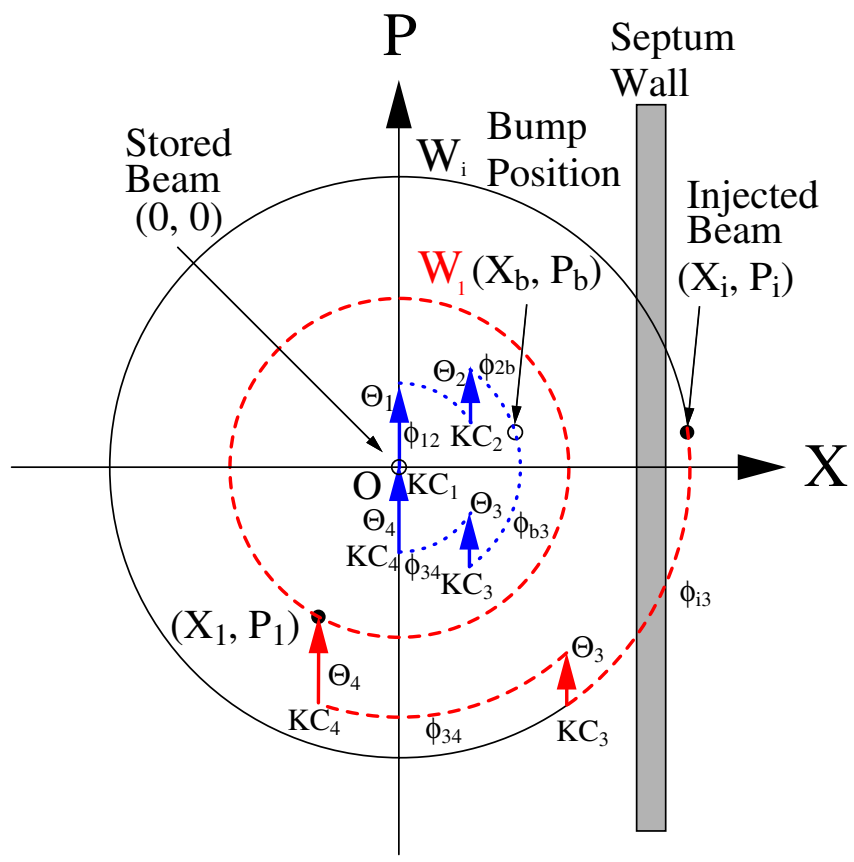

FIG. 4. (Color) Same as Fig. 3, but the trajectories are generated by four kickers to give the closed local bump. The closed and open circles indicate the injected and stored beam, and the dashed and dotted lines show their trajectories, respectively. 
coordinate of $\left(X_{1}, P_{1}\right)$ of the reduced invariant. The trajectory of the injected beam is shown by the dashed line, as shown in Fig. 4. Therefore, for the injected beam, the downstream kickers $K C_{3}$ and $K C_{4}$ are important in order to obtain the reduced invariant.

For the closed bump, the kick angles are calculated under the following conditions: both the entrance and exit coordinates are $(0,0)$ and the coordinate of $\left(X_{b}, P_{b}\right)$ exists between $\mathrm{KC}_{3}$ and $\mathrm{KC}_{4}$. The normalized kick angles of four kickers are $\Theta_{i}(i=1,2,3,4)$, and they are given by

$$
\left\{\begin{array}{l}
\Theta_{1}=\frac{1}{\sin \phi_{12}}\left(X_{b} \cos \phi_{2 b}-P_{b} \sin \phi_{2 b}\right), \\
\Theta_{2}=-\frac{1}{\sin \phi_{12}}\left\{X_{b} \cos \left(\phi_{12}+\phi_{2 b}\right)-P_{b} \sin \left(\phi_{12}+\phi_{2 b}\right)\right\}, \\
\Theta_{3}=-\frac{1}{\sin \phi_{34}}\left\{X_{b} \cos \left(\phi_{b 3}+\phi_{34}\right)+P_{b} \sin \left(\phi_{b 3}+\phi_{34}\right)\right\}, \\
\Theta_{4}=\frac{1}{\sin \phi_{34}}\left(X_{b} \cos \phi_{b 3}+P_{b} \sin \phi_{b 3}\right),
\end{array}\right.
$$

where $\phi_{i j}=\phi_{j}-\phi_{i}$ is the phase advance from $\phi_{i}$ to $\phi_{j}$, which corresponds to the location of the kickers and the bump. In the case of four kickers, we can generate the closed local bump of an arbitrary position and angle.

As shown in Fig. 5, when both the phase advances $\phi_{1 b}$ and $\phi_{b 2}$ between the injection point and the location of the kickers are $90^{\circ}$, only two kickers allow us to generate the closed bump and $P_{b}$ equals zero. Then, the kick angles are simply given by $\Theta_{1}=\Theta_{2}=X_{b}$, and the reduced invariant $W_{1}$ is calculated to be $\Delta X^{2}=\left(X_{i}-X_{b}\right)^{2}$, where the coordinates of the injection point are $\left(X_{i}, P_{i}=0\right)$. In the twobump method, the phase advance between the kickers and

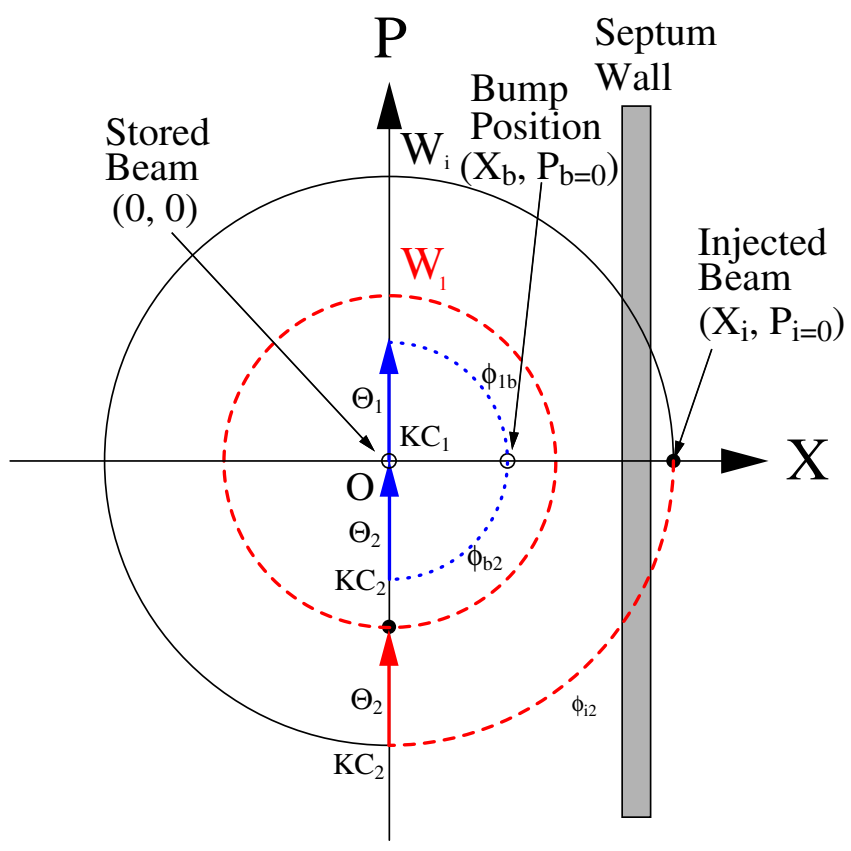

FIG. 5. (Color) Same as Fig. 4, but the trajectories generated by two kickers that are located at a special phase advance of $\pi$ are represented. the injection point is severely limited to close the bump; however, we have an economic advantage: we can reduce the number of the expensive kicker magnets.

By using the two-bump method, we can easily understand the unclosed-bump technique. When the kick angle $\Theta_{2}$ of the upstream kicker $K C_{2}$ is larger than the kick angle $\Theta_{1}$ of the downstream kicker $K C_{1}$, i.e., $\Theta_{2}>\Theta_{1}$, we can further reduce the invariant of the injected beam compared with the closed bump while the coherent dipole oscillation is generated in the stored beam. When the kick angles $\Theta_{1}$ and $\Theta_{2}$ are given by

$$
\left\{\begin{array}{l}
\Theta_{1}=X_{b}, \\
\Theta_{2}=(1+\alpha) \Theta_{1}=(1+\alpha) X_{b}, \quad(\alpha>0),
\end{array}\right.
$$

where $\alpha$ is an enhancement factor, the reduced invariant $W_{1, u}$ of the injected beam and the produced invariant $W_{s, u}$ of the stored beam are expressed by

$$
\left\{\begin{aligned}
W_{1, u} & =\left(X_{i}-\Theta_{2}\right)^{2}=\left\{X_{i}-(1+\alpha) X_{b}\right\}^{2}, \\
& >\left(X_{i}-X_{b}\right)^{2}=W_{1, c} \\
W_{s, u} & =\left(\Theta_{2}-\Theta_{1}\right)^{2}=\left(\alpha X_{b}\right)^{2}>0
\end{aligned}\right.
$$

where $W_{1, c}$ is the reduced invariant for the closed bump. If the produced invariant $W_{s, u}$ is acceptable, the intentional unclosed-bump method is effective to obtain much smaller invariant $W_{1, u}$ than that of the closed bump $W_{1, c}$. In practice, it appears that the enhanced factor $\alpha$ of 0.1 to 0.2 is adequate. This technique is available in the case of the four-bump method.

\section{B. Injection scheme using a single PQM}

Next, we formulate the injection scheme using a single $\mathrm{PQM}$ and derive its strength to realize the beam injection in electron storage rings. Here, we assume that the PQM provides a single kick to the injected beam until next injection. The beam is also treated as a single particle. This injection scheme is similar to that by using the twobump method. However, since the quadrupole magnet has a zero field at the center of its magnetic pole, the stored beam is not kicked when it passes through the central position. Thus, the single PQM enables us to inject the beam into the ring because upstream magnets are not required for the stored beam. In addition, since the orbit of the stored beam is preserved on the central orbit even if the PQM is excited, we only need to treat the formulation of the injected beam motion.

The orbit of the injected beam follows a circle $W_{i}$, as shown in Fig. 6 . When $x_{i}^{\prime}=-\left(\alpha_{x, i} / \beta_{x, i}\right) x_{i}, P_{i}$ becomes zero and $W_{i}$ assumes the minimum value of $X_{i}^{2}$, and the normalized injection position is simply expressed by

$$
X_{i}=\sqrt{W_{i}},
$$

when $X_{i}$ is positive. The PQM is located at the normalized coordinates $\left(X_{0}, P_{0}\right)$ from where the injected beam passes 


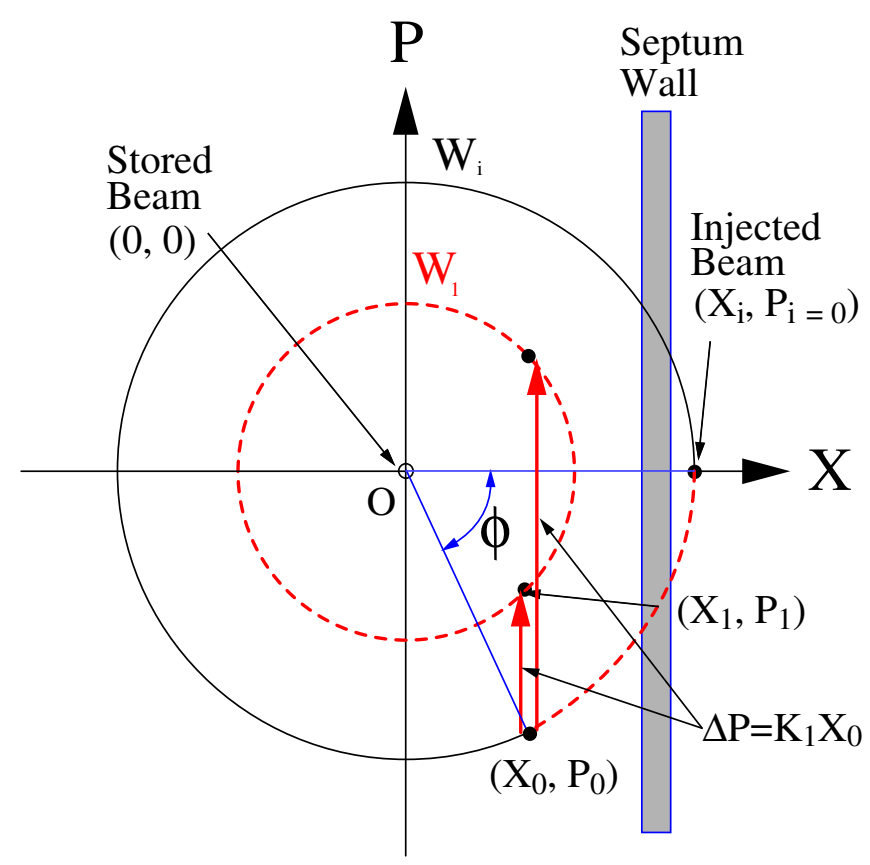

FIG. 6. (Color) Same as Fig. 4, but using a single PQM instead of several kickers for the beam injection. $\phi$ denotes the phase advance between the injection point and the location of the PQM, and $K_{1}$ represents the normalized strength of the PQM. The dashed line shows the trajectory of the injected beam.

during the first turn. When the phase advance between the location of the PQM and the injection point is $\phi$, The elements of the normalized coordinate are represented by

$$
\left\{\begin{array}{l}
X_{0}=X_{i} \cos \phi=\sqrt{W_{i}} \cos \phi \\
P_{0}=-X_{i} \sin \phi=-\sqrt{W_{i}} \sin \phi
\end{array}\right.
$$

where $\phi$ rotates in the clockwise direction. Since the PQM also provides a kick to the beam, only the normalized angle is changed. When the amount of change is $\Delta P$, new normalized coordinates $\left(X_{1}, P_{1}\right)$ are given by

$$
\left\{\begin{array}{l}
X_{1}=X_{0}=\sqrt{W_{i}} \cos \phi, \\
P_{1}=P_{0}+\Delta P=-\sqrt{W_{i}} \sin \phi+\Delta P,
\end{array}\right.
$$

and the new circle $W_{1}$ is expressed by

$$
W_{1}=X_{1}^{2}+P_{1}^{2}=W_{i}-2 \Delta P \sqrt{W_{i}} \sin \phi+\Delta P^{2},
$$

as shown in Fig. 6. This $W_{1}$ becomes the reduced invariant of the injection beam after the PQM. Then, $\Delta P$ is obtained by solving Eq. (13). Using $W_{i}, W_{1}$, and $\phi$, it is given by

$$
\Delta P=-\sqrt{W_{i}} \sin \phi \mp \sqrt{W_{1}-W_{i} \cos ^{2} \phi} .
$$

Here, we represent $\Delta P$ using the normalized focusing strength $K_{1}$ in the thin-lens approximation,

$$
\left(\begin{array}{l}
X_{1} \\
P_{1}
\end{array}\right)=\left(\begin{array}{cc}
1 & 0 \\
-K_{1} & 1
\end{array}\right)\left(\begin{array}{l}
X_{0} \\
P_{0}
\end{array}\right)
$$

and then

$$
\Delta P=-K_{1} X_{0}=-K_{1} \sqrt{W_{i}} \cos \phi,
$$

where $K_{1}>0$ indicates a focusing quadrupole magnet in the horizontal direction. As a result, the strength becomes

$$
\begin{aligned}
K_{1} & =\frac{-\sqrt{W_{i}} \sin \phi \mp \sqrt{W_{1}-W_{i} \cos ^{2} \phi}}{\sqrt{W_{i}} \cos \phi} \\
& =-\tan \phi \mp \sqrt{\left(\frac{W_{1}}{W_{i}}\right) \frac{1}{\cos ^{2} \phi}-1 .}
\end{aligned}
$$

The strength $K_{1}$ depends on the phase advance and the reduction ratio $r$, which is defined by

$$
r=\frac{W_{1}}{W_{i}}
$$

In order to make the beam injection easier, the goal of the $\mathrm{PQM}$ injection scheme is to make the ratio $r$ lower. However, this has to be done without making $K_{1}$ too large. In addition, there is a restriction on where the PQM can positioned: it has to be positioned away from where the injection trajectory crosses the beam axis.

We numerically examine the behavior of $K_{1}$ as a function of $\phi$ and $r$. Figure 7 shows $K_{1}$, which is calculated by substituting some proper values of $r$ in Eq. (17). We provide the values of $r$ from 0.2 to 0.5 in steps of 0.1 . The horizontal axis shows $\phi$ from $40^{\circ}$ to $140^{\circ}$, and the vertical axis shows $K_{1}$ from -0.4 to $0.4 . K_{1}$ is a doublevalued function of $\phi$. The sign in Eq. (17) that produces the smaller absolute value of $K_{1}$ will be taken as the desired solution for $\phi$, as shown in Fig. 7 and others. From Fig. 7 , we can determine some important aspects of the behavior of $K_{1} . K_{1}$ is limited in some ranges of $\phi$, and the range becomes narrow with a decrease in $r$. In addition, $K_{1}$ increases with a decrease in $r$. This indicates that it is

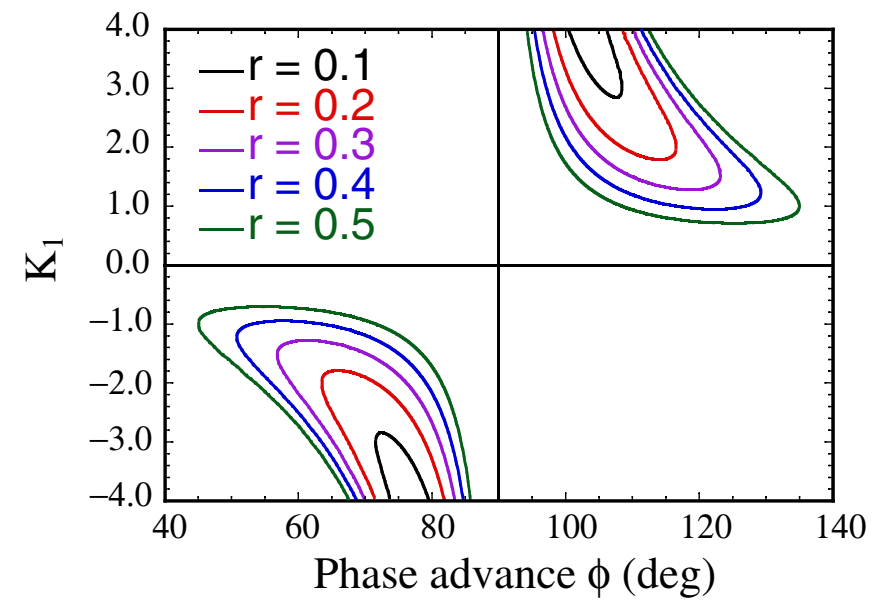

FIG. 7. (Color) Normalized strength $K_{1}$ is displayed as a function of the phase advance $\phi$ from the injection point and reduction ratio $r$. 
TABLE I. Main parameters of the PF-AR at an injection energy of $3.0 \mathrm{GeV}$. The Twiss parameters at the injection point are included.

\begin{tabular}{lcc}
\hline \hline \multicolumn{1}{c}{ Parameters } & Symbol & Value \\
\hline Injection beam energy & $E$ & $3.0 \mathrm{GeV}$ \\
Circumference & $C$ & $377.26 \mathrm{~m}$ \\
Betatron tunes & $\nu_{x}, \nu_{y}$ & $10.15,10.19$ \\
Revolution period & $T_{\mathrm{rev}}$ & $1.257 \mu \mathrm{s}$ \\
Horizontal emittance & $\varepsilon_{x}$ & $63.0 \mathrm{~nm} \mathrm{rad}$ \\
Vertical emittance & $\varepsilon_{y}$ & $0.63 \mathrm{~nm} \mathrm{rad}$ \\
rf voltage & $V_{\mathrm{rf}}$ & $3.5 \mathrm{MV}$ \\
Radiation damping time & $\tau_{x}, \tau_{y}, \tau_{\varepsilon}$ & $25.0,25.0,12.5 \mathrm{~ms}$ \\
Energy spread & $\sigma_{\varepsilon}$ & 0.00053 \\
Bunch length & $\sigma_{z}$ & $10.385 \mathrm{~mm}$ \\
Horizontal beta function & $\beta_{x, i}$ & $20.48 \mathrm{~m}$ \\
Horizontal alpha function & $\alpha_{x, i}$ & -3.257 \\
Vertical beta function & $\beta_{y, i}$ & $5.568 \mathrm{~m}$ \\
Vertical alpha function & $\alpha_{y, i}$ & 0.435 \\
Horizontal dispersion function & $\eta_{x, i}$ & $0.480 \mathrm{~m}$ \\
Horizontal dispersion function derivative & $\eta_{x, i}^{\prime}$ & -0.012 \\
\hline \hline
\end{tabular}

difficult to obtain a small reduced invariant because we require a large strength of the PQM. For $\phi$ close to $90^{\circ}$ the beam passes through the quadrupole near its center, where the quadrupole field is zero. Thus, there is little kick possible. As a result, we recognize that it is essential to locate the $\mathrm{PQM}$ in the range of $\phi$ from $57^{\circ}$ to $123^{\circ}$ from the injection point in order to obtain $r$ less than 0.3. Further, the solutions corresponding to the choice of $\phi$ require that the normalized $K_{1}$ with an absolute value of more than 1.3. In practice, we require a physical strength $k_{1}$ or an integrated field gradient $B^{\prime} \ell$ to produce the magnet. The $k_{1}$ is expressed by

$$
k_{1}=\frac{K_{1}}{\beta_{x, 1}}
$$

where $\beta_{x, 1}$ is the horizontal betatron function at the PQM. $B^{\prime} \ell$ is given using $k_{1}$ as

$$
B^{\prime} \ell=k_{1} B \rho,
$$

where $B^{\prime}$ is the field gradient of the magnet; $\ell$, the magnetic length, and $B \rho$, the magnetic rigidity. For example, $k_{1}$ is calculated to be $0.065 \mathrm{~m}^{-1}$ when $K_{1}$ is 1.3 and $\beta_{x, 1}$ is $20 \mathrm{~m}$; then, $B^{\prime} \ell$ is estimated to be $0.65 \mathrm{~T}$ at a beam energy of $3.0 \mathrm{GeV} . \beta_{x, 1}$ depends on the lattice configuration and focusing system of the ring, and it becomes a function of $\phi$. Therefore, as a next step, we apply the formulation of the injection scheme using the PQM to the PF-AR, which is an existing machine, and obtain the optimized value of $k_{1}$ to realize the beam injection in the PF-AR.

\section{Application to the PF-AR}

The PF-AR was originally constructed as a booster synchrotron for the TRISTAN, which was the electronpositron collider. The main parameters of the PF-AR are listed in Table I. The lattice consists of four arc sections, four long straight sections of length greater than $20 \mathrm{~m}$, and eight short straight sections of length $5.5 \mathrm{~m}$. The injection point is located at one of the short straight sections in the south region of the ring. The lattice configuration and optical functions around the injection point are shown in Fig. 8. The coordinate at the injection point of the PF-AR is

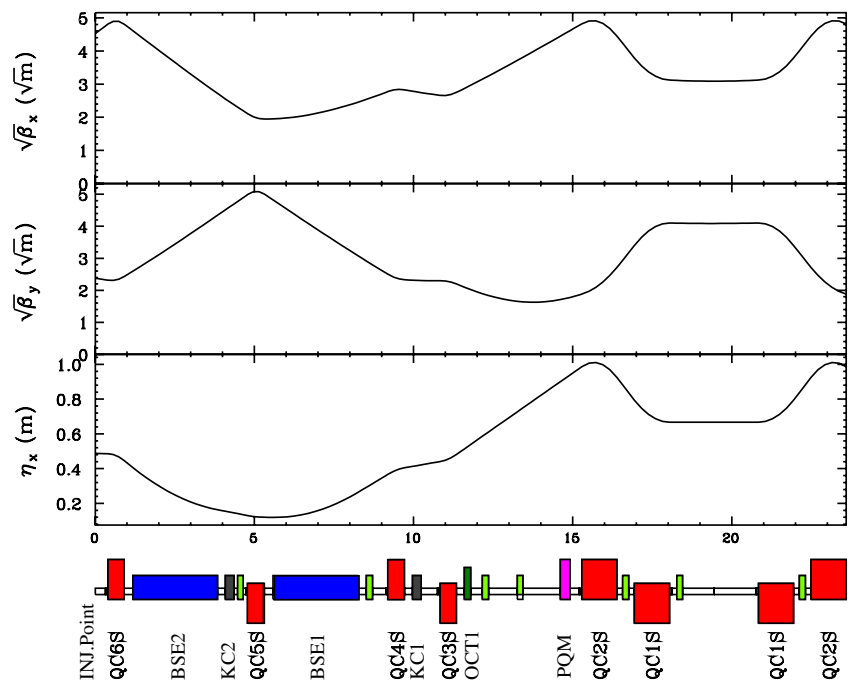

FIG. 8. (Color) Lattice configurations and optical functions of the PF-AR around the injection point. The upper, middle, and lower parts show the square root of the horizontal betatron function $\sqrt{\beta_{x}}$, square root of the vertical betatron function $\sqrt{\beta_{y}}$, and horizontal dispersion function $\eta_{x}$, respectively. The rectangular boxes indicate bending magnets (BSE2 and BSE1), quadrupole magnets (QC6S, QC5S, QC4S, QC3S, QC2S, and QC1S), octupole magnets (OCT1), kicker magnets (KC1 and $\mathrm{KC} 2$ ), and a pulsed quadrupole magnet (PQM). 
designed to be $\left(x_{i}, x_{i}^{\prime}\right)=(48.0 \mathrm{~mm}, 7.63 \mathrm{mrad})$. The Twiss parameters at the point are $\alpha_{x, i}=-3.257$ and $\beta_{x, i}=$ $20.48 \mathrm{~m}$, the normalized coordinate is $\left(X_{i}, P_{i}\right)=$ $(10.61,0.00)$; thus, the injection invariant $W_{i}$ is calculated to be $W_{i}=112.5 \mathrm{~mm}$ mrad. The acceptance of the ring in the horizontal direction is estimated to about $60 \mathrm{~mm}$ mrad. We have four kicker magnets to form a pulsed local bump with the coordinate of $\left(x_{b}, x_{b}^{\prime}\right)=(22.0 \mathrm{~mm}, 3.50 \mathrm{mrad})$. The reduced invariant $W_{1}$ is obtained to be $33.0 \mathrm{~mm}$ mrad, which corresponds to $r$ of about 0.3 . We assume that we can achieve beam injection with a single PQM if we can obtain $r$ of less than 0.3 by the PQM.

Based on Eqs. (17) and (18), the required $k_{1}$ is calculated as a function of the path length $s$ from the injection point. Figure 9 shows required $k_{1}$ as a function of $s$ depending on the six values of $r$ from 0.178 to 0.355 . Here, $\phi$ is estimated by

$$
\phi=\tan ^{-1}\left(\frac{P_{0}(s)}{X_{0}(s)}\right)=\tan ^{-1}\left(\alpha_{x, 0}(s)+\beta_{x, 0}(s) \frac{x_{0}^{\prime}(s)}{x_{0}(s)}\right),
$$

where $x_{0}(s)$ and $x_{0}^{\prime}(s)$ are the position and divergence angle of the injected beam, respectively; $\beta_{x, 0}$ and $\alpha_{x, 0}$ are the Twiss parameters at a path length $s$ from the injection point. We observe that the locations that yield $r$ less than 0.3 are limited to those between about 7 and $18 \mathrm{~m}$ from the injection point, which correspond to $\phi$ between $57^{\circ}$ and $123^{\circ}$, respectively. For the $r$ values considered, $k_{1}$ is smaller at around $16 \mathrm{~m}$ from the injection point, as indicated by the rectangular region in Fig. 9. This region is enlarged and shown with the quadrupole magnets in Fig. 10. Unfortunately, the best location for PQM is inside the quadrupole magnet $\mathrm{QC} 2 \mathrm{~S}$, so we select instead a location about $0.3 \mathrm{~m}$ upstream of QCS2; at this location we require $k_{1}$ of more than $0.07 \mathrm{~m}^{-1}$ in order to obtain $r$ of less than 0.3 .

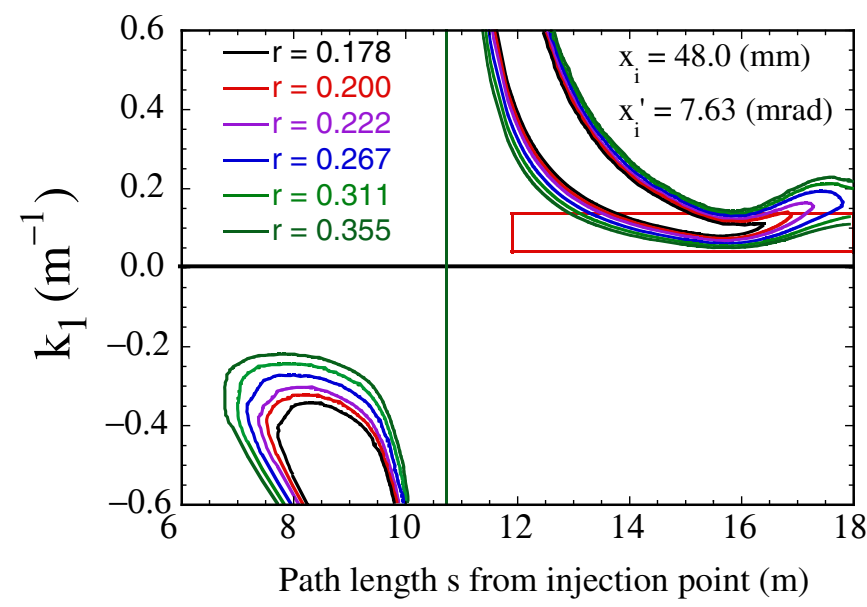

FIG. 9. (Color) Physical strength $k_{1}$ as a function of the path length $s$ from the injection point and reduction ratio $r$.

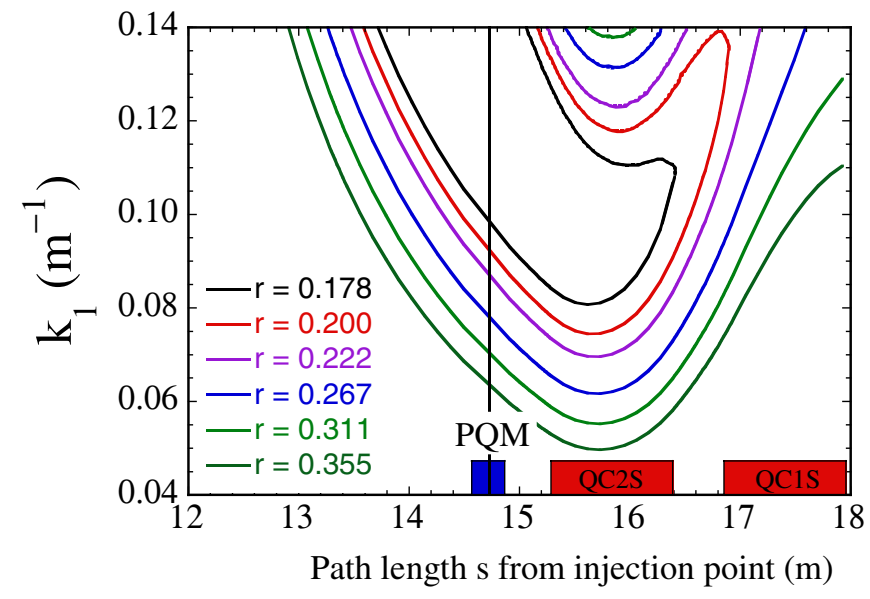

FIG. 10. (Color) Same as Fig. 10, but the rectangular region in Fig. 10 is enlarged and the quadrupole magnets QC1S and QC2S and the PQM are shown together. The perpendicular solid line indicates the center of the PQM.

Thus, the required location and strength of the PQM are calculated from the formulation of the beam injection scheme using the PQM in the PF-AR.

\section{SIMULATION OF BEAM INJECTION USING A SINGLE PQM}

\section{A. For the injected beam}

In the formulation, we have treated the barycenter of the injected beam as a single particle and the particle motion is assumed to be linear by neglecting the nonlinearities in the ring. However, from a practical viewpoint, we must consider the beam sizes and include the nonlinearities, which mainly arise from the sextupole magnets used for the chromaticity correction and the octupole magnets used for suppressing the transverse instabilities in the PF-AR. In order to evaluate these effects, we conducted a multiparticle tracking simulation in which we provided the distributions of the particles in the injected beam as an initial condition. We employed the simulation code SAD (strategic accelerator design), which is developed in High Energy Accelerator Research Organization (KEK) [7]. The injected beam is represented by 1000 macroparticles with Gaussian distributions in the six-dimensional phase space $\left(x-x^{\prime}, y-y^{\prime}, z-\delta\right)$, where $\delta$ indicates an energy deviation from the designed energy. Table II lists the simulation parameters of the injected beam.

Figure 11 shows the normalized phase-space plots in the horizontal direction with the injected beam, the beam just before the kick, the beam just after the kick by the PQM, and the circulated beams for five turns for the six values of $k_{1}$, namely, $0.05,0.07,0.09,0.11,0.13$, and $0.15 \mathrm{~m}^{-1}$. The red and blue circles indicate the reduced and injected invariants of 33.0 and $112.5 \mathrm{~mm}$ mrad compared with the results of the simulation, respectively. The vertical solid lines indicate the physical apertures normalized by the 
TABLE II. Initial conditions of the injected beam for a multiparticle tracking simulation.

\begin{tabular}{lcc}
\hline \hline \multicolumn{1}{c}{ Parameter } & Symbol & Value \\
\hline Macroparticle number & $N_{\text {part }}$ & 1000 \\
Tracking turn number & $N_{\text {turn }}$ & 200 \\
Horizontal emittance & $\varepsilon_{x}$ & $150 \mathrm{~nm} \mathrm{rad}$ \\
Vertical emittance & $\varepsilon_{y}$ & $150 \mathrm{~nm} \mathrm{rad}$ \\
Energy spread & $\sigma_{\varepsilon}$ & 0.00125 \\
Bunch length & $\sigma_{z}$ & $1.0 \mathrm{~mm}$ \\
Horizontal injection position & $x_{i}$ & $48.0 \mathrm{~mm}$ \\
Horizontal injection angle & $x_{i}^{\prime}$ & $7.63 \mathrm{mrad}$ \\
Vertical injection position & $y_{i}$ & $0.00 \mathrm{~mm}$ \\
Vertical injection angle & $y_{i}^{\prime}$ & $0.00 \mathrm{mrad}$ \\
Longitudinal injection position & $z_{i}$ & $0.00 \mathrm{~mm}$ \\
Beam energy deviation & $\delta$ & 0.00 \\
\hline \hline
\end{tabular}

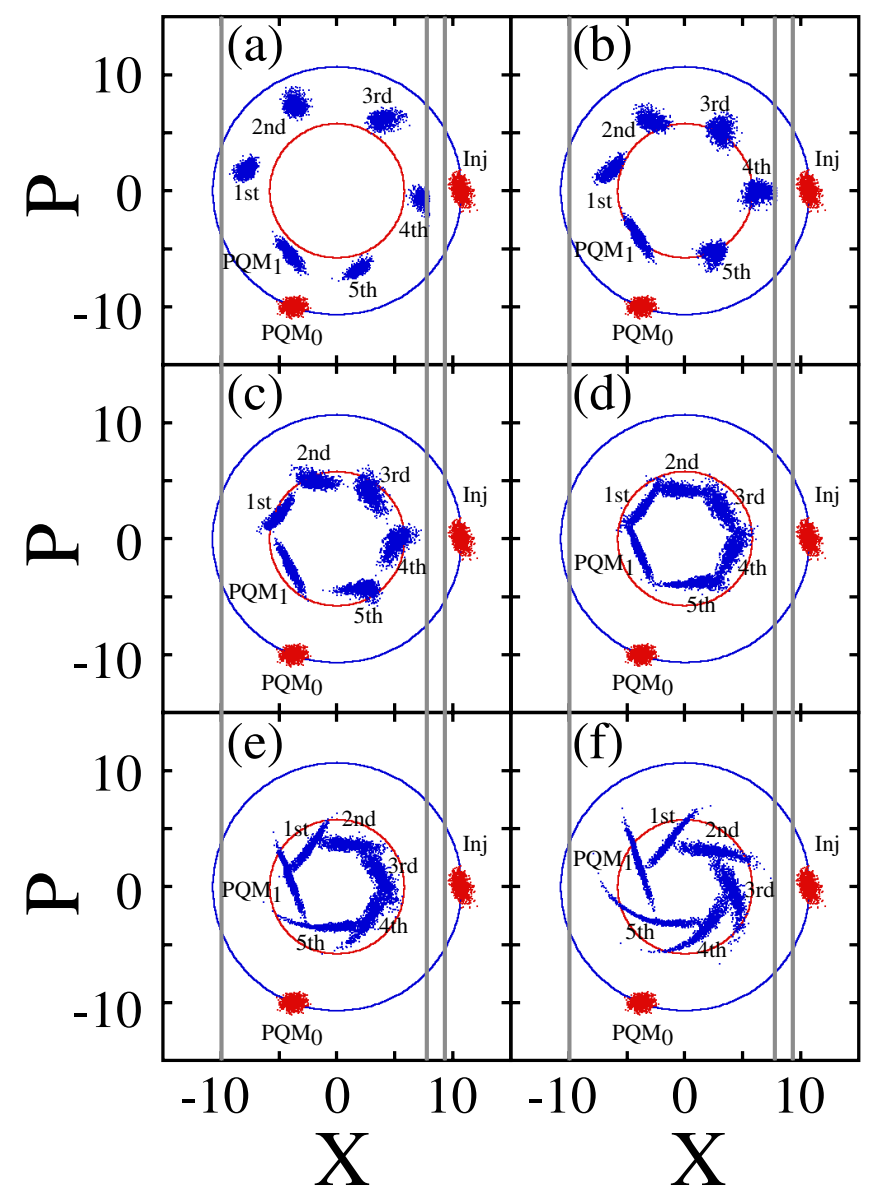

FIG. 11. (Color) Normalized horizontal phase-space plots of the injected beam and the circulated beam after the kick until five turns are displayed for the normalized strengths $K_{1}$ of (a) 0.989 , (b) 1.384, (c) 1.780, (d) 2.175 , (e) 2.571, and (f) 2.967, which correspond to the physical strengths $k_{1}$ of $0.05,0.07,0.09,0.11$, 0.13 , and $0.15 \mathrm{~m}^{-1}$, respectively. The beam just before the kick due to the PQM is also represented by $\mathrm{PQM}_{0}$ and $\mathrm{PQM}_{1}$, respectively. Three solid lines indicate normalized horizontal physical apertures. The blue and red solid circles represent the injection invariant $W_{i}$ of $112.5 \mathrm{~mm} \mathrm{mrad}$ and the reduced invariant $W_{1}$ of $33.0 \mathrm{~mm} \mathrm{mrad}$, respectively. betatron function at the injection point, which correspond to the narrowest horizontal aperture in the PF-AR. The physical apertures are included in the simulation. In order to obtain the reduced invariant of $33 \mathrm{~mm} \mathrm{mrad}$, the physical strength $k_{1}$ of $0.07 \mathrm{~m}^{-1}$ is required from the formulation described in the previous section. As shown in Fig. 11(b), it appears that $k_{1}$ of $0.07 \mathrm{~m}^{-1}$ is slightly small considering the beam size of the injected beam since the circulated beam is very close to the aperture at the 4th turn. In the case of the smaller $k_{1}$ of $0.05 \mathrm{~m}^{-1}$, a beam loss occurs at the 4 th turn. The simulation results indicate that $k_{1}$ more than $0.09 \mathrm{~m}^{-1}$ is suitable.

Next, in order to examine the effects of the nonlinearities in the ring, we carried out the particle tracking simulations for 200 turns. Figure 12 shows the horizontal phase-space plots at the injection point at turn 200 of the injected beam.

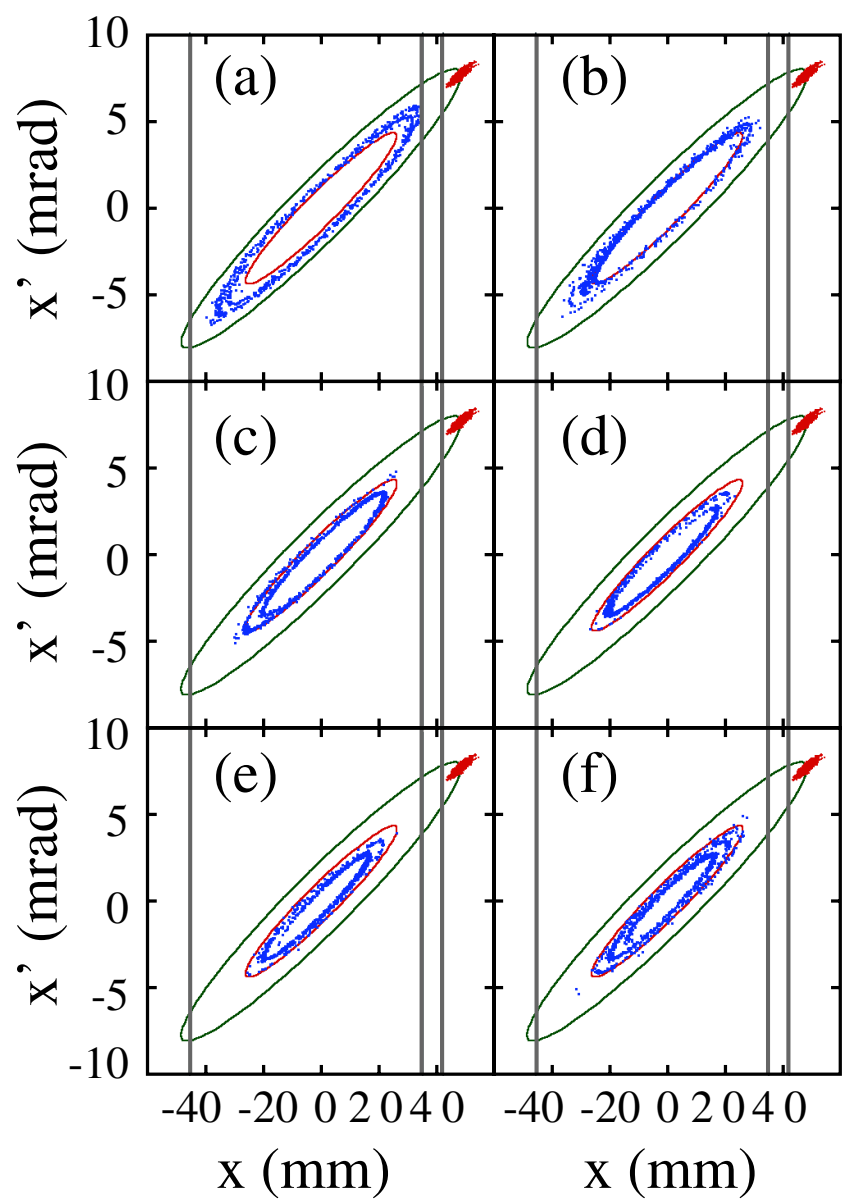

FIG. 12. (Color) Horizontal phase-space plots of the circulated beam at turn number 200 at the injection point are displayed for the physical strengths $k_{1}$ of (a) 0.05, (b) 0.07, (c) 0.09, (d) 0.11, (e) 0.13 , and (f) $0.15 \mathrm{~m}^{-1}$. The injected beam is also represented. Three solid lines indicate horizontal physical apertures, which correspond to the vacuum chamber at the injection point. The green and red solid ellipses represent the injection invariant $W_{i}$ of $112.5 \mathrm{mmmrad}$ and the reduced invariant $W_{1}$ of $33.0 \mathrm{~mm}$ mrad, respectively. 
Since the beam is smeared by the effects of the nonlinearities, the particles are spread in the phase space. The effects are not very strong; therefore, all particles survive the 200 turns when $k_{1}$ is more than $0.09 \mathrm{~m}^{-1}$. In the cases in which $k_{1}$ exceeds $0.09 \mathrm{~m}^{-1}$, the injected beam may be captured in the acceptance of the ring without a beam loss. However, the beam loss is observed in the cases in which $k_{1}$ is $0.07 \mathrm{~m}^{-1}$ since the reduced invariant for the lost particles is slightly larger than the physical aperture. The beam losses are estimated to be $15 \%$ and $0.2 \%$ during the 200 turns for $k_{1}$ of 0.05 and $0.07 \mathrm{~m}^{-1}$, respectively. Thus, the simulation results indicate $k_{1}$ should be larger than $0.09 \mathrm{~m}^{-1}$ in order to avoid the beam loss as much as possible in the beam injection using the PQM.

Although we have not mentioned the optics matching between the beam transport and the ring until now, the rematching may help the beam injection of the PQM scheme because it is going through the new quadrupole for the injected beam.

\section{B. For the stored beam}

Here, we examine the effect of the PQM on the stored beam. When the stored beam is treated as a single particle, it is not influenced by the PQM and it passes through the central position of the magnet. However, since the real beam has a distribution, most of the particles are influenced by the PQM even if the barycenter of the beam passes through the central position. Therefore, we also simulated the stored beam using the multiparticle tracking method.

The initial conditions of the stored beam used in the simulation are listed in Table I. The stored beam is excited by the PQM with $k_{1}$ of $0.09 \mathrm{~m}^{-1}$. Figure 13 shows the horizontal and vertical beam sizes $\sigma_{x}$ and $\sigma_{y}$, respectively, as a function of the turn number until 50 turns. $\sigma_{x}$ and $\sigma_{y}$ are calculated as standard deviations for the Gaussian distributions projected in each of the horizontal and vertical directions. $\sigma_{x}$ and $\sigma_{y}$ at the central location of the north long straight section without the kick denoted by $\sigma_{x, 0}$ and $\sigma_{y, 0}$ are stable at 0.494 and $0.049 \mathrm{~mm}$, respectively. However, at $k_{1}=0.09 \mathrm{~m}^{-1}, \sigma_{x}$ and $\sigma_{y}$ are modulated by the PQM: $\sigma_{x}$ varies from 0.3 to $1.2 \mathrm{~mm}$, while $\sigma_{y}$ slightly varies from 0.047 to $0.053 \mathrm{~mm}$. As a result, $\sigma_{x}$ is strongly changed by the excitation of the PQM with $k_{1}=$ $0.09 \mathrm{~m}^{-1}$, and the variation from a smaller to a larger size is estimated to be over 2.4 times larger as compared with the beam size before the kick. In the single PQM scheme, the modulation in the beam profile of the stored beam cannot be avoided. This may be a disadvantage for top-up operation.

\section{HARDWARE DESCRIPTION OF THE PQM SYSTEM}

In this section, we describe the design consideration of the PQM system, which consists of a pulsed magnet,

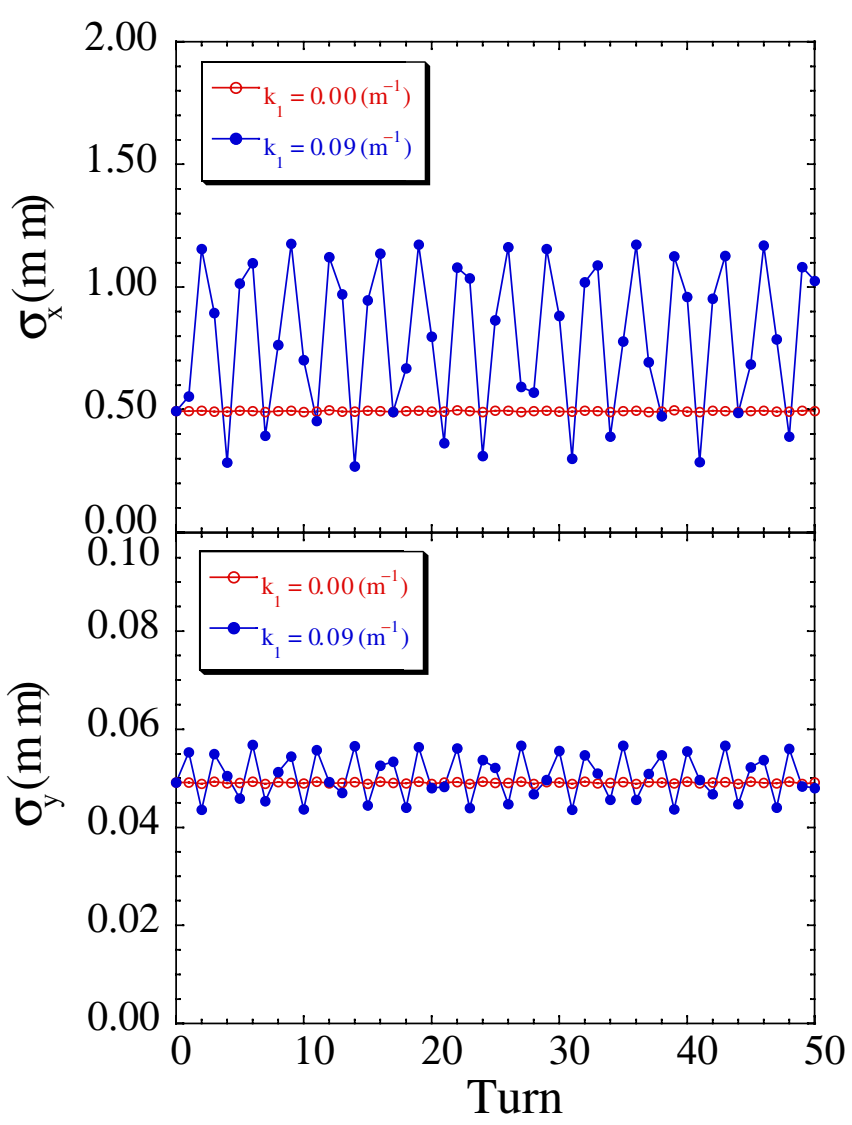

FIG. 13. (Color) Horizontal and vertical beam sizes, $\sigma_{x}$ and $\sigma_{y}$, are represented as a function of the turn number. The closed and open circles show the beam sizes after a kick with the physical strength $k_{1}$ of $0.09 \mathrm{~m}^{-1}$ and without a kick $\left(k_{1}=0.00 \mathrm{~m}^{-1}\right)$. The beam sizes of the stored beam at the central location of the north long straight section without the kick, $\sigma_{x, 0}$ and $\sigma_{y, 0}$, are constant at 0.494 and $0.049 \mathrm{~mm}$, respectively. $\sigma_{x}$ is modulated by the PQM and varies from 0.3 to $1.2 \mathrm{~mm}$, while $\sigma_{y}$ slightly varies from 0.047 to $0.053 \mathrm{~mm}$.

pulsed power supply, and ceramic chamber. In addition, we describe the result of field measurements carried out on the PQM.

\section{A. Pulsed quadrupole magnet}

First, a core length $\ell$ of $0.3 \mathrm{~m}$ was determined in consideration of free space around the candidate location where the PQM will be installed in the PF-AR. Then, the magnet with a field gradient $B^{\prime}$ of more than $3 \mathrm{~T} / \mathrm{m}$ at a beam energy of $3.0 \mathrm{GeV}$ was required from Eq. (20) based on the result of the simulation that $k_{1}$ of more than $0.09 \mathrm{~m}^{-1}$ is necessary for the beam injection. Next, we evaluated the aperture of the ring to determine the gap or the bore diameter of the magnet. It is relatively easy to obtain a higher field gradient in a quadrupole magnet with a smaller gap while such a gap may limit the physical aperture of the ring. The aperture in the vertical direction is fixed by the vacuum chamber with a vertical inner size of 


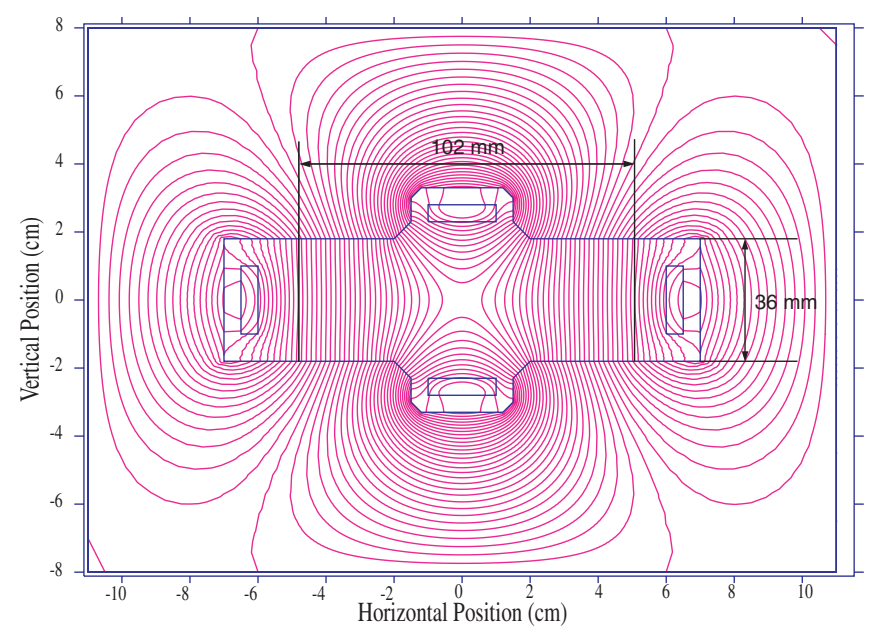

FIG. 14. (Color) Cross-sectional view of the PQM with magnetic field lines calculated by the two-dimensional code POISSON. The horizontal and vertical gaps are 102 and $36 \mathrm{~mm}$, respectively. The maximum field strength at a current of $2000 \mathrm{~A}$ is about $0.2 \mathrm{~T}$ at the locations near upper and lower coils.

$20 \mathrm{~mm}$ for the insertion device (ID-NE1) located in the north long straight section. The vertical beta functions at the central location of the ID-NE1 and at the location of the PQM are 3.1 and $3.9 \mathrm{~m}$, respectively. Thus, a vertical inner size of more than $23 \mathrm{~mm}$ was required for the vacuum chamber of the PQM so that the vertical aperture is not narrowed. In general, a ceramic chamber is employed for the pulsed magnet, which is operated at a speed of the order of microseconds. The chamber requires a thickness of more than $5 \mathrm{~mm}$ and a clearance of more than $1 \mathrm{~mm}$ for the installation of the magnet. The vertical gap is determined to be $36 \mathrm{~mm}$ by adding $23 \mathrm{~mm}$ to the sum of the chamber thickness and clearance. The horizontal gap is

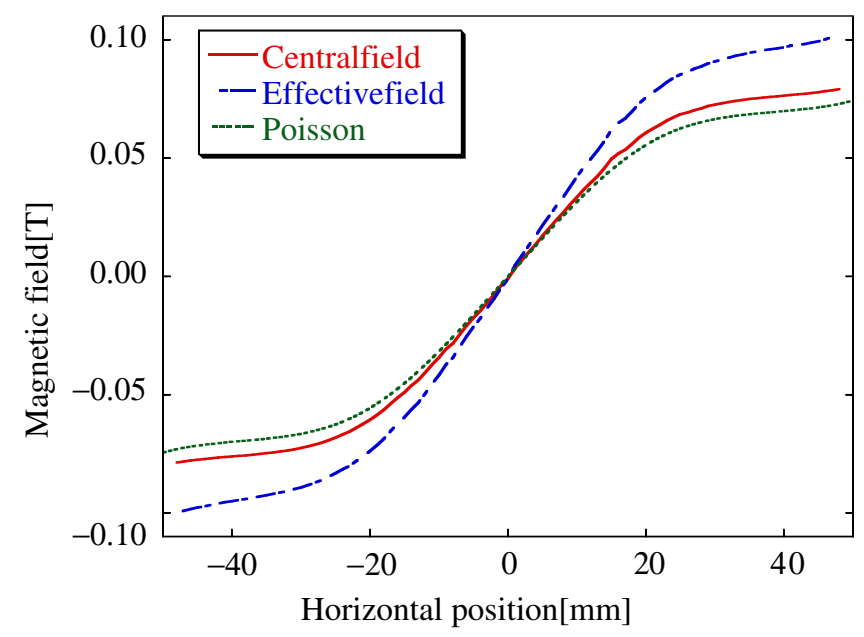

FIG. 15. (Color) Calculated and measured field strengths of the $\mathrm{PQM}$ as a function of the horizontal position. The lines represent the measured central field strength, measured effective field strength, and calculated field strength.
TABLE III. Specifications of the PQM system.

\begin{tabular}{lcc}
\hline \hline Parameter & Symbol & Value \\
\hline Field gradient & $B^{\prime}$ & $3 \mathrm{~T} / \mathrm{m}$ \\
Current & $I$ & $2000 \mathrm{~A}$ \\
Voltage & $V$ & $10.5 \mathrm{kV}$ \\
Horizontal gap & $g_{h}$ & $102 \mathrm{~mm}$ \\
Vertical gap & $g_{v}$ & $36 \mathrm{~mm}$ \\
Core length & $\ell$ & $0.30 \mathrm{~m}$ \\
Calculated inductance & $L$ & $4 \mu \mathrm{H}$ \\
Pulse width & $\tau$ & $2.4 \mu \mathrm{s}$ \\
\hline \hline
\end{tabular}

also determined to be $102 \mathrm{~mm}$ based on a similar calculation.

A Panofsky-type magnet [8] with a single-turn coil was adopted to reduce the inductance of the magnet and suppress the voltage of the power supply; the estimated inductance was about $3 \mu \mathrm{H}$. Figure 14 shows a cross-sectional view of the $\mathrm{PQM}$ with magnetic force line distributions calculated by the two-dimensional code POISSON [9]. The horizontal distribution of the magnetic field strength is shown in Fig. 15. The field calculation confirmed that a field gradient of $3 \mathrm{~T} / \mathrm{m}$ was achieved at an excitation current of $2000 \mathrm{~A}$. The magnet was constructed from 0.15 -mm-thick lamination silicon steel in order to minimize the eddy current effects. $0.15 \mathrm{~mm}$ was the minimum thickness that we could easily obtain. The processing precision of the steel was less than $0.2 \mathrm{~mm}$. The magnet was manufactured by the Japanese company, IDX Co., Ltd.

In order to prevent a complex process by multiple kicks during several turns, the pulse width less than $2.4 \mu$ s that is twice the revolution period of the PF-AR was required. The specifications of the PQM are listed in Table III.

\section{B. Pulsed power supply}

We adopted a command charging power supply consisting of two parts: one part is a DC high-voltage power supply for charging, and the other part is a pulser with two thyratron switches for forming a current pulse. Figure 16 shows the conceptual block diagram of the circuit for the power supply. The circuit is designed as an LCR resonant circuit. The capacitor is charged by driving the first thyratron switch (E2V Technologies CX1159); then, the charge stored in the capacitor goes to an inductive load on driving the second thyratron switch (E2V Technologies CX1174). We employed two trigger signals to drive the thyratron switches; the second discharging trigger signal is sent after more than $10 \mathrm{~ms}$ from the first charge trigger signal.

The inductive load consists of the PQM of $3 \mu \mathrm{H}$ and the connection cable of $1 \mu \mathrm{H}$. The total inductance $L$ is estimated to be $4 \mu \mathrm{H}$. While the pulse is rising, the circuit functions as an LC circuit. The full period $\tau$ of the pulse is $4.8 \mu \mathrm{s}$ and the main capacitance $C$ is calculated to be $0.12 \mu \mathrm{F}$. Then, the charging voltage $V$ is estimated to be 


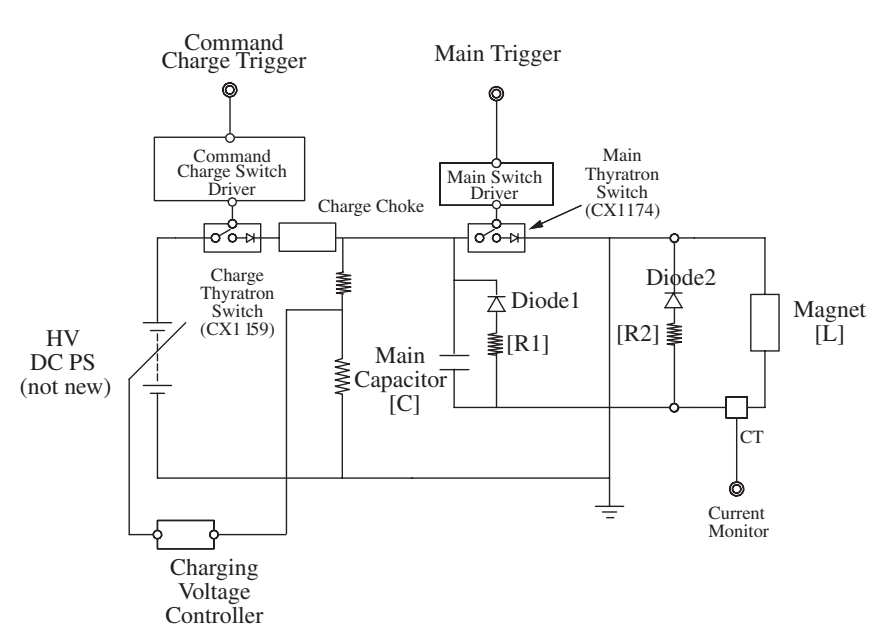

FIG. 16. Conceptual block diagram of the circuit for the pulsed power supply. The power supply consists of two parts: a DC part and a pulser part. Two thyratron switches in the pulser part are used for the charge and the discharge switches. They are driven by the independent trigger signals. The magnet serves as an inductive load.

about $10.5 \mathrm{kV}$ at a current of $2000 \mathrm{~A}$. When the charge comes back from the load, it passes through a diode, and then the circuit functions as an overdamped LCR circuit. Further, by adjusting the values of resistors $R_{1}$ and $R_{2}$, the reflection current is sufficiently suppressed and the pulse width of $2.4 \mu \mathrm{s}$ is achieved. The power supply can be operated at the maximum repetition rate of $25 \mathrm{~Hz}$, which corresponds to the repetition rate of the beam injection for the PF-AR. It was also manufactured by IDX Co., Ltd.

We intended to install the pulser at a neighboring location in the accelerator tunnel without protection for a radiation damage. Hence, the cable length between the $\mathrm{PQM}$ and the pulser was assumed to be about $1 \mathrm{~m}$. However, before the installation we estimated that the radiation damage for the pulser was too severe. In order to avoid the radiation damage the pulser was installed outside of the accelerator tunnel, and a 10-m connection cable was used.

\section{Ceramic chamber}

In the case of a metallic vacuum chamber, the eddy currents produced due to the rapid variation of the magnetic field cause distortion and delay of the magnetic field. To avoid these problems, we used a ceramic chamber, as shown in Fig. 17. It has a rectangular inner aperture of $90 \mathrm{~mm}$ in width and $24 \mathrm{~mm}$ in height. The thickness of the chamber was $5 \mathrm{~mm}$. Its inner side was coated with a molybdenum-titanium (Mo-Ti) layer of $3 \mu \mathrm{m}$ thickness to reduce the beam-coupling impedance. Fe-Ni-Co alloys were deposited at both ends of the ceramic chamber, which was connected with steel special use stainless (SUS) chambers. An absorber mask of copper $(\mathrm{Cu})$ was also attached in the upstream SUS chamber to prevent synchrotron radiation from impinging on the chamber. The temperature of

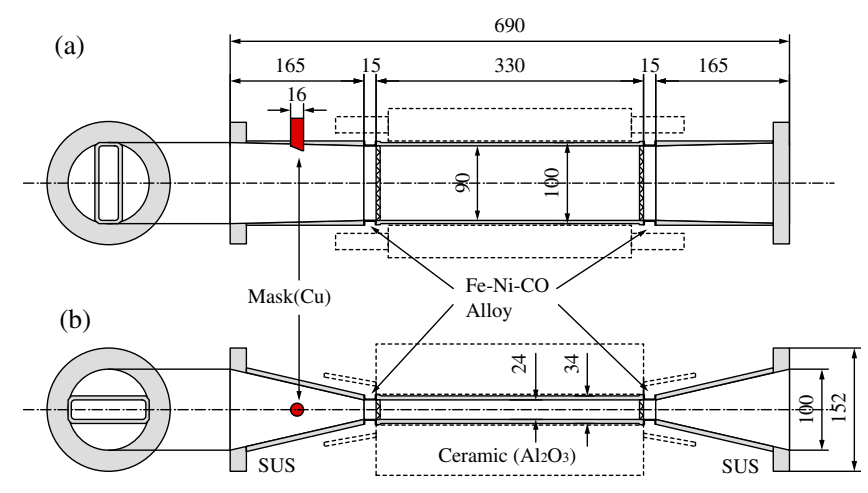

FIG. 17. (Color) Ceramic chamber for the PQM: (a) top view and (b) side view of the chamber. The inner aperture of the chamber is rectangular; it is $90 \mathrm{~mm}$ in width by $24 \mathrm{~mm}$ in height. The thickness of the chamber is $5 \mathrm{~mm}$. The outer aperture is rectangular; it is $100 \mathrm{~mm}$ in width by $34 \mathrm{~mm}$ in height. The inner side of the chamber is coated with a molybdenum-titanium (MoTi) layer of $3 \mu \mathrm{m}$ thickness, and an absorber mask of copper $\mathrm{Cu}$ is also attached. The dashed line indicates the PQM.

the chamber did not increase much even when a singlebunch beam of $60 \mathrm{~mA}$ was stored in the ring.

\section{Field measurements}

After the pulsed quadrupole magnet, the ceramic chamber, and the power supply were manufactured, the magnetic fields were measured using search coils. In order to measure the central field and integral field simultaneously, a pair of coils were employed: a short coil of $5 \mathrm{~mm} \times$ $5 \mathrm{~mm}$ size for the central field and a long coil of $5 \mathrm{~mm} \times$ $60 \mathrm{~cm}$ size for the integral field. Each coil is made of a single-turn fine copper wire attached to a glass epoxy bar. The voltage induced in the search coil is expressed by

$$
V=-\frac{d \phi}{d t}=-S \frac{d B}{d t}, \quad B_{\text {peak }}=-\frac{1}{S} \int_{0}^{\text {peak }} V d t,
$$

where $\phi$ is the magnetic flux penetrating the search coil, $S$ is the coil area, and $B$ is the magnetic field density. The peak value of the magnetic field $B_{\text {peak }}$ was calculated by integrating the induced voltage.

Data was acquired using a digital oscilloscope (Tektronix TDS748) connected with an online computer through general purpose interface board (GP-IB). The power supply was also remotely controlled through the GP-IB relay. To produce a timing signal for the charging and discharging switches, a pulse generator (Agilent 33220A) was employed. The typical waveform measured at a current of $2000 \mathrm{~A}$ is shown in Fig. 18. Line Ch1 shows a voltage from the short coil, line $C h 2$ shows that from the current transfer of the power supply, and line $C h 3$ represents that from the long coil. The voltage induced in the long coil reached about $0.6 \mathrm{kV}_{\mathrm{p}-\mathrm{p}}$. The horizontal distribution of the measured field is shown in Fig. 15. The magnetic field is close to zero near the pole center and it 


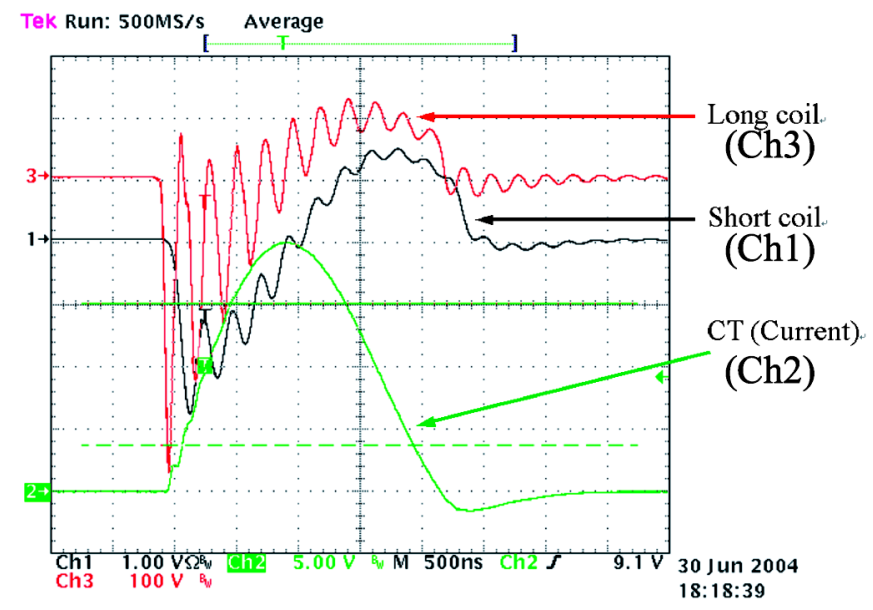

FIG. 18. (Color) Typical waveform obtained in a field measurement using a digital oscilloscope. The horizontal axis shows time; each division is of $50 \mathrm{~ns}$. The lines represent the output signals from the long search coil on the vertical axis with $100-\mathrm{V}$ divisions (Ch3: red), the short search coil on the vertical axis with $1-\mathrm{V}$ divisions (Ch1: black), and the current transformer on the vertical axis with $5-\mathrm{V}$ divisions (Ch2: green), respectively.

is as a function of the horizontal position near zero. The field measurements confirmed that the magnet clearly worked as a quadrupole magnet with gradient of $3 \mathrm{~T} / \mathrm{m}$ at a current of $2000 \mathrm{~A}$ and a voltage of $12 \mathrm{kV}$. The voltage was slightly larger than the predicted voltage of $10.5 \mathrm{kV}$. The inductance of the system was estimated to be $4.6 \mu \mathrm{H}$. Since the power supply has the capacity of more than $24 \mathrm{kV}$ for the voltage, we attempted to achieve twice the

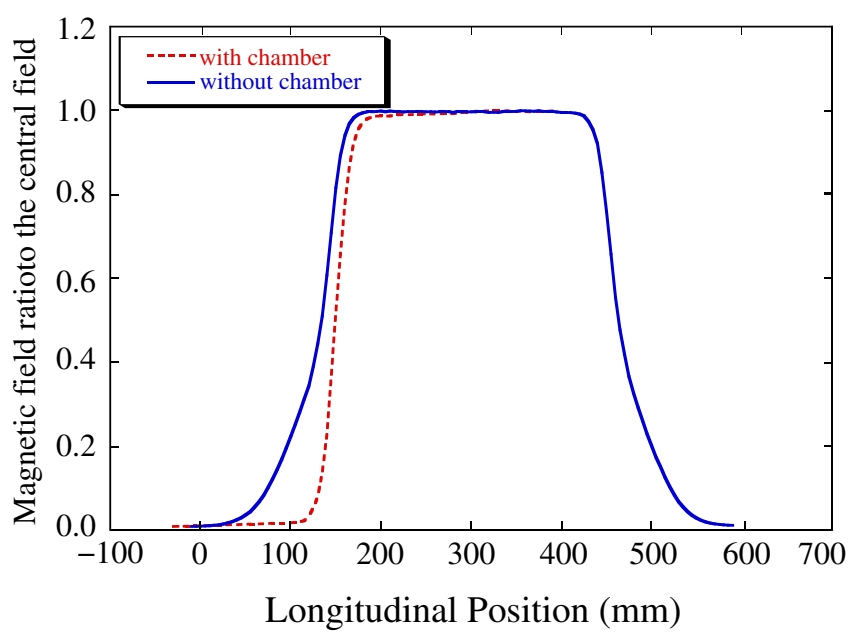

FIG. 19. (Color) Field strength distribution measured at the horizontal position of $15 \mathrm{~mm}$ from the pole center as a function of the longitudinal position. The measurement is carried out using a short coil. The blue solid and red dotted lines represent the measured results with and without a vacuum chamber, respectively. Since the chamber is almost symmetric, the measurement with the chamber is carried out on only one side of the magnet. designed current. We achieved a current of about $4000 \mathrm{~A}$, which corresponds to $k_{1}$ of $0.18 \mathrm{~m}^{-1}$, at a repetition rate of $25 \mathrm{~Hz}$.

The longitudinal field distribution measured along the horizontal position of $15 \mathrm{~mm}$ from the pole center is displayed in Fig. 19. The effective field strength was slightly reduced due to the SUS chambers attached to both the sides of the ceramic chamber. However, the effective field gradient was sufficient even with the reduction.

The magnet itself and the girder were charged due to the induced voltage from the pulsed field. A ground connection was very important to prevent the induced voltage from destroying the instruments or giving an electric shock to a human being. Therefore, the magnet, the girder, and the vacuum chamber were connected to a low impedance metal plate at the ground potential; this plate was located inside the accelerator tunnel.

\section{EXPERIMENTAL RESULT USING A REAL BEAM}

The experiment using a real beam was conducted after installing the PQM. For the beam injection, we first synchronized the excitation timing of the PQM with the timing of the injected beam. The excitation timing is the discharge switch timing of the pulsed power supply. The timing signal was generated by the injection trigger signal from the injector linac. Since the trigger signal comes before about $100 \mu \mathrm{s}$ of the beam, a delay of about $100 \mu \mathrm{s}$ was added to the trigger signal. A fine-tuning of the timing was achieved by controlling a delay module of the order of nanoseconds by comparing the signal from the PQM current transformer with the signal from the ring current

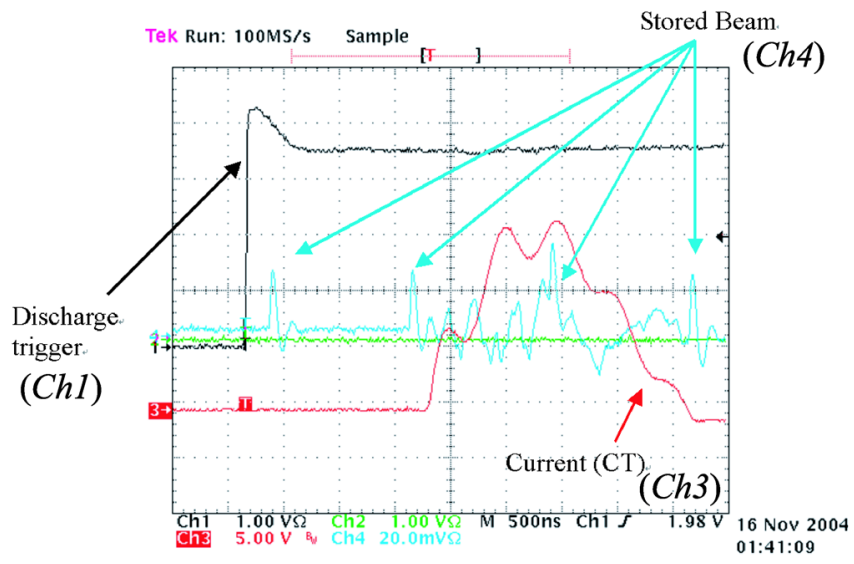

FIG. 20. (Color) Typical waveform in the adjustment of excitation timing of the PQM measured using a digital oscilloscope. The lines represent a discharge trigger signal ( $C h 1$ : black), a signal from the current transformer of the ring ( $C h 4$ : cyan), and a signal from the current transformer of the PQM power supply (Ch3: red). The peaks in (Ch4: cyan) line indicate the stored bunch. 


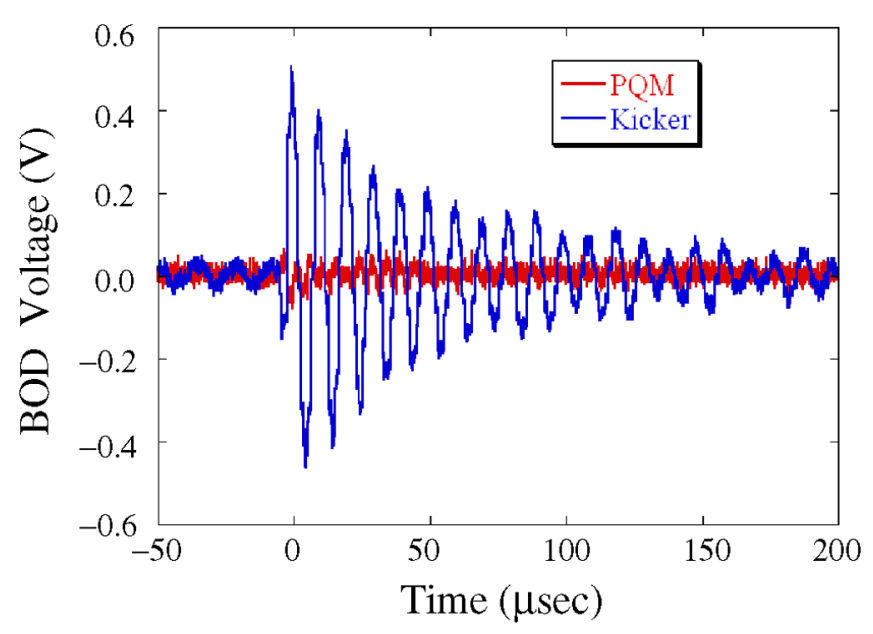

FIG. 21. (Color) Typical coherent dipole oscillation of the stored beam in the horizontal direction measured using a beam detection monitor just after the injection. The output voltage of the monitor is displayed as a function of time. The dipole oscillation after the excitation of the PQM (red solid line) is greatly reduced as compared with that after the excitation of the kickers (blue dashed line).

transformer, as shown in Fig. 20. The pulse shape modulation of the PQM current transformer was generated due to the impedance mismatch, since the pulser was installed outside of the accelerator tunnel and a 10-m connection cable was used instead of a 1-m cable. In practice, this was not a serious problem for the single PQM injection scheme.

As a result, we succeeded in storing the beam using the single PQM and the stored beam current gradually increased. At this time, however, an injection rate $r_{\text {inj }}$ was quite low. $r_{\text {inj }}$ is defined by

$$
r_{\mathrm{inj}}=\frac{d I}{d t},
$$

where $I$ is the stored current and $t$ is the injection time.

Next, we corrected the orbit of the stored beam around the PQM to make the beam pass through a center of the field. A horizontal local bump of about $1 \mathrm{~mm}$ was required to achieve this, but a vertical local bump was not required. Figure 21 shows the horizontal oscillations of the stored beam detected using the beam oscillation detector (BOD), which enables us to measure the dipole oscillations turn by turn. The dipole oscillation after the excitation of the PQM was greatly reduced as compared with that after the excitation of the kickers. Various tuning operations improved $r_{\text {inj }}$ gradually.

Figure 22 shows $r_{\text {inj }}$ as a function of $k_{1}$. The measurements were conducted with a repetition rate of $25 \mathrm{~Hz}$ and a constant injected beam current. When $k_{1}$ is less than $0.06 \mathrm{~m}^{-1}, r_{\text {inj }}$ is near zero. As $k_{1}$ increases from 0.06 to $0.11 \mathrm{~m}^{-1}, r_{\text {inj }}$ gradually increases and reaches the maximum value of $1.0 \mathrm{~mA} / \mathrm{s}$, which is comparable with the value obtained for the beam injection using the kickers. $r_{\mathrm{inj}}$

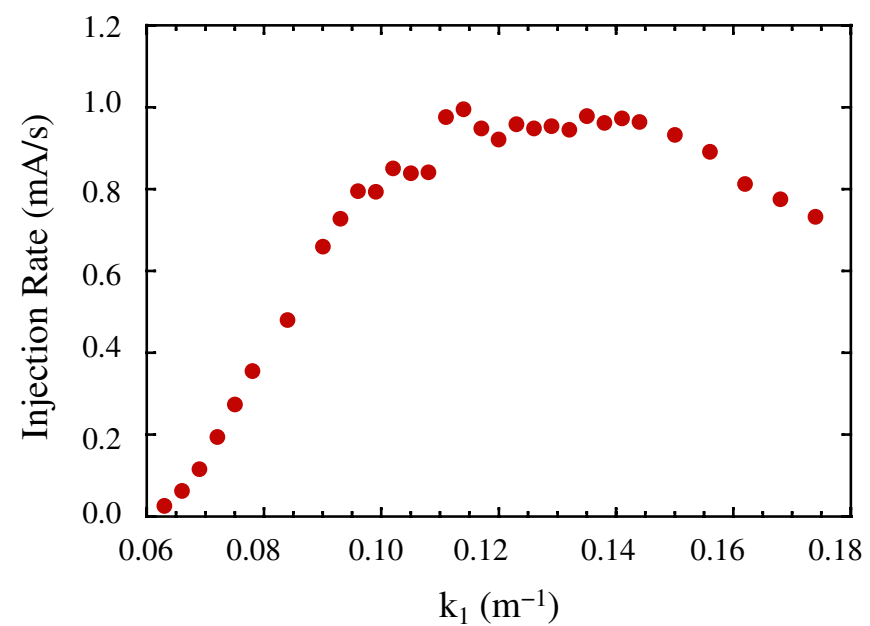

FIG. 22. (Color) Injection rates are shown as a function of the physical strength $k_{1} . k_{1}$ ranges from 0.06 to $0.18 \mathrm{~m}^{-1}$. The rates are calculated from straightline fits over $20 \mathrm{~s}$.

has a constant value of about $0.95 \mathrm{~mA} / \mathrm{s}$ between 0.11 and $0.14 \mathrm{~m}^{-1}$; then, it gradually decreases as $k_{1}$ exceeds $0.14 \mathrm{~m}^{-1}$. When $k_{1}$ is $0.09 \mathrm{~m}^{-1}$, which is the designed strength, $r_{\text {inj }}$ is about $0.7 \mathrm{~mA} / \mathrm{s}$. This is $30 \%$ smaller than the maximum value of $1.0 \mathrm{~mA} / \mathrm{s}$ obtained by $k_{1}$ with $0.11 \mathrm{~m}^{-1}$. Though $0.11 \mathrm{~m}^{-1}$ may be suitable in the PFAR beam injection, the behavior of $r_{\text {inj }}$ qualitatively agrees with the prediction of the beam loss estimated by the multiparticle tracking simulations.

After this optimization, we attempted to realize a beam current as high as possible. Figure 23 shows the data when we realized the maximum current of $68 \mathrm{~mA}$. However, it was necessary to adjust the rf voltage and other parameters in order to obtain a higher beam current by overcoming the strong beam instabilities that occur when the beam injec-

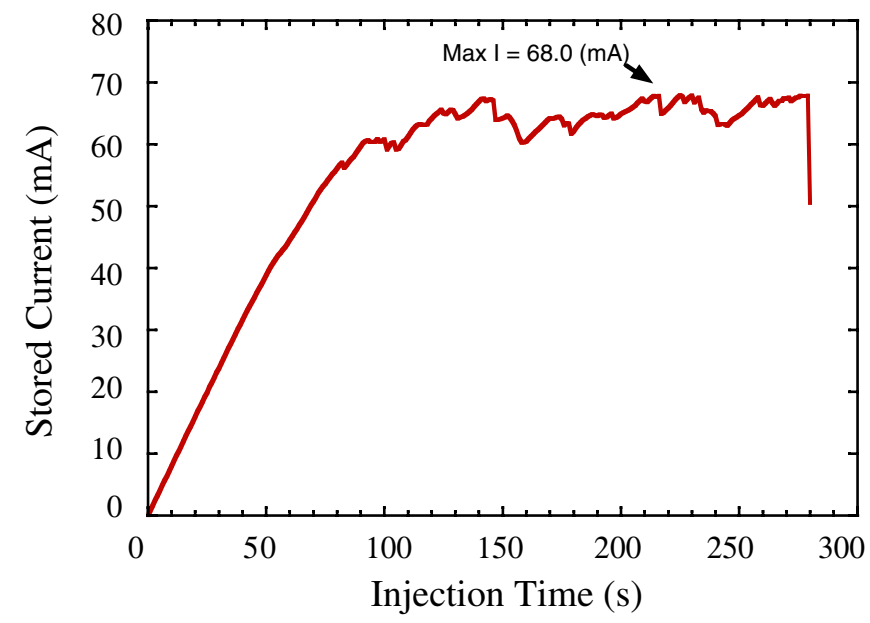

FIG. 23. (Color) Stored current when a maximum current of $68 \mathrm{~mA}$ is realized. Various tuning operations are required to increase the limit due to strong beam instabilities from 60 to $68 \mathrm{~mA}$. 
tion is done with the kickers. It was hoped that the beam instabilities would be reduced from not having the stored beam kicked. We have continued to examine the effects on the injected and stored beam due to the PQM in the experiment. More experimental results, including the conflicts with the instabilities, will be presented in another paper.

\section{CONCLUSION}

In the PF-AR, we demonstrated a new injection scheme using a single PQM, which allows us to store the beam without using a pulsed local bump in electron storage rings. We developed the PQM system, which consists of a pulsed magnet, pulsed power supply, and ceramic chamber. The field measurement confirmed the specifications: the field gradient of $3 \mathrm{~T} / \mathrm{m}$ at the excitation current of $2000 \mathrm{~A}$, and the pulse width of $2.4 \mu \mathrm{s}$. After installing the PQM into the PF-AR tunnel, the experiment using a real beam was conducted. Then, we succeeded in injecting the beam into the ring using the PQM and storing the beam at a current of more than $60 \mathrm{~mA}$. The injection rate is as good as that using the pulsed local bump when the physical strength $k_{1}$ is set to be between 0.11 and $0.14 \mathrm{~m}^{-1}$. This is the first successful beam injection using a single PQM in electron storage rings. Since the principle of the new injection scheme is very simple, it can apply to most electron storage rings.

\section{ACKNOWLEDGMENTS}

The authors greatly thank T. Obina, K. Umemori, C. Weixing, and T. Mitsuhashi for their help in conducting the experiment. They wish to thank Y. Hori, Y. Tanimoto, and $\mathrm{T}$. Uchiyama for producing and installing the ceramic chamber. Further, they thank the other staff of the light source division of the Photon Factory and the staff of the Accelerator Facility in KEK for the ring operation. They also wish to thank M. Kobayashi, previous director of the light source division, for his advice and support. Finally, they wish to thank T. Kasuga, present director of the light source division, for his continuous encouragement.

[1] See, for example, Gottfried Mülhaupt, Synchrotron Radiation Sources A Primer, Chapter 3: Injection Systems (World Scientific, Singapore, 1994).

[2] H. Tanaka, T. Ohsima, K. Soutome, and M. Takao, Nucl. Instrum. Methods Phys. Res., Sect. A 539, 547 (2005).

[3] T. Abe, K. Ebihara, E. Ezura, K. Haga, K. Harada, Y. Hori, T. Ieiri, S. Isagawa, T. Kageyama, T. Kasuga, T. Katoh, H. Kawata, M. Kikuchi, M. Kobayashi, Y. Kobayashi, K. Kudo, Y. Minagawa, T. Mitsuhashi, T. Miyajima, S. Nagahashi, T. T. Nakamura, H. Nakanishi, T. Nogami, T. Obina, Y. Ohsawa, Y. Onishi, M. Ono, T. Ozaki, H. Sakai, Y. Sakamoto, S. Sakanaka, M. Sato, M. Satoh, T. Shioya, M. Suetake, R. Sugahara, M. Tadano, T. Takahashi, S. Takasaki, Y. Takeushi, Y. Tanimoto, M. Tejima, K. Tsuchiya, T. Uchiyama, A. Ueda, K. Umemori, N. Yamamoto, S. Yamamoto, M. Yoshioka, and T. Fujita, in AIP Conference Proceedings of Eighth International Conference on Synchrotron Radiation Instrumentation (AIP, San Francisco, California, 2003), Vol. 705, pp. 33-36.

[4] K. Hanaoka, Y. Funakoshi, H. Hayano, T. Higo, and K. Satoh, Nucl. Instrum. Methods Phys. Res., Sect. A 329, 16 (1993).

[5] T. Fujita, T. Kasuga, Y. Minagawa, and T. Obina, in Proceedings of the 2003 Particle Accelerator Conference (IEEE, Portland, Oregon, 2003), pp. 32613263.

[6] K. Harada, Y. Kobayashi, T. Miyajima, and S. Nagahashi, in Proceedings of the 3rd Asian Particle Accelerator Conference (Pohang Accelerator Laboratory, Gyeongju, Korea, 2004), pp. 344-346.

[7] Strategic Accelerator Design, http://acc-physics.kek.jp/ SAD.

[8] Y. Li, P Chin, R. Kishek, M. Reiser, M. Venturini, J. G. Wang, Y. Zou, and T.F. Godlove, in Proceedings of the 1999 Particle Accelerator Conference (IEEE, New York, NY, 1999), pp. 3369-3371.

[9] POISSON SUPERFISH, Los Alamos National Laboratory Report No. LA-UR-96-1834 (revision 14 March 2000). 